

\title{
Moments and One level density of certain unitary families of Hecke $L$-functions
}

\author{
par PENG GAO et LIANGYI ZHAO
}

\begin{abstract}
RÉSumÉ. Dans cet article, nous étudions les moments des valeurs centrales de certaines familles unitaires de fonctions $L$ de Hecke sur le corps des rationnels de Gauss et prouvons un résultat quantitatif de non-annulation de leurs valeurs centrales. Nous établissons aussi un résultat de densité portant sur les petits zéros dans ces familles de fonctions $L$ de Hecke.
\end{abstract}

ABSTRACT. In this paper, we study the moments of central values of certain unitary families of Hecke $L$-functions of the Gaussian field, and establish quantitative non-vanishing result for their central values. We also establish a one level density result for the low-lying zeros of these families of Hecke $L$-functions.

\section{Introduction}

The non-vanishing of central values of $L$-functions is of central importance in number theory. In the classical case of Dirichlet $L$-functions, S. Chowla [5] conjectured that $L(1 / 2, \chi) \neq 0$ for every primitive Dirichlet character $\chi$. One typical way to investigate this non-vanishing problem is to study the moments of a family of $L$-functions. By considering the first and second mollified moments of $L(1 / 2, \chi)$, B. Balasubramanian and V. K. Murty [1] showed that $L(1 / 2, \chi) \neq 0$ for at least $4 \%$ of Dirichlet characters $\chi \bmod q$. For primitive characters, the proportion was improved to $1 / 3$ by H. Iwaniec and P. Sarnak in [13], to $34.11 \%$ by H. M. Bui [4] and most recently to $3 / 8$ by R. Khan and H. T. Ngo [16].

Instead of mollified moments, one may be only interested in the moments of Dirichlet $L$-functions. The first moment of the family of primitive Dirichlet $L$-functions of modulus $q$ has long been known while the second moment is due to R. E. A. C. Paley [20]. In [10], D. R. Heath-Brown obtained an asymptotic formula for the fourth moment of the family of $L$-functions associated with primitive Dirichlet characters modulo $q$, provided $q$ does not have too many distinct prime divisors. The formula was extended to

Manuscrit reçu le 4 août 2018, révisé le 16 janvier 2020, accepté le 12 décembre 2020.

Mathematics Subject Classification. 11M41, 11L40.

Mots-clefs. Hecke $L$-functions, Hecke characters.

P. G. is supported in part by NSFC grant 11371043 and 11871082 and L. Z. by the FRG grant PS43707 and the Faculty Silverstar Award PS49334. 
all integers by K. Soundararajan in [23]. An asymptotic formula for prime moduli with power savings was obtained by M. P. Young in [25] and this result was later improved by V. Blomer, E. Fouvry, E. Kowalski, P. Michel and D. Milićević [3].

As an analogue of Dirichlet $L$-functions, T. Stefanicki [24] obtained the first and second moments of Dirichlet twists of modular $L$-functions. The formula for the second moment is valid for a density zero set and is extended to almost all integers in [6].

Motivated by the result of Stefanicki, we consider in this paper a family of Hecke $L$-functions in the Gaussian field. Throughout this paper, we let $K=\mathbb{Q}(i)$ and $\mathcal{O}_{K}=\mathbb{Z}[i]$ for the ring of integers in $K$. We also denote $U_{K}=\langle i\rangle$ for the group of units in $\mathcal{O}_{K}$. Let $q \in \mathcal{O}_{K}$ with $(q, 2)=1$ and $\chi$ be a homomorphism:

$$
\chi:\left(\mathcal{O}_{K} /(q)\right)^{*} \rightarrow S^{1}:=\{z \in \mathbb{C}|| z \mid=1\} .
$$

We shall say $\chi$ is a character modulo $q$. Note that in $\mathcal{O}_{K}$, every ideal coprime to 2 has a unique generator congruent to 1 modulo $(1+i)^{3}$ (see the paragraph above Lemma 8.2.1 in [2]). Such a generator is called primary. When $q$ is co-prime to $2, \chi$ induces a character $\tilde{\chi}$ modulo $(1+i)^{3} q$. To see this, note that the ring $\left(\mathcal{O}_{K} /(1+i)^{3} q\right)^{*}$ is isomorphic to the direct product of the group of units $U_{K}$ and the group $N_{q}$ formed by elements in $\left(\mathcal{O}_{K} /(1+i)^{3} q\right)^{*}$ and congruent to $1\left(\bmod (1+i)^{3}\right)$ (i.e., primary). Under this isomorphism, any element $n \in\left(\mathcal{O}_{K} /(1+i)^{3} q\right)^{*}$ can be written uniquely as $n=u_{n} \cdot n_{0}$ with $u_{n} \in U_{K}, n_{0} \in N_{q}$. We can now define $\widetilde{\chi}\left(\bmod (1+i)^{3} q\right)$ such that for any $n \in\left(\mathcal{O}_{K} /(1+i)^{3} q\right)^{*}$,

$$
\widetilde{\chi}(n)=\chi\left(n_{0}\right)
$$

We say that $\chi$ is a primitive character modulo $q$ if it does not factor through $\left(\mathcal{O}_{K} /\left(q^{\prime}\right)\right)^{*}$ for any proper divisor $q^{\prime}$ of $q$. When $\chi$ is primitive and $\chi(-1)=$ -1 , we will show in Section 2.1 that the character $\tilde{\chi}$ is also primitive modulo $(1+i)^{3} q$. As $\tilde{\chi}$ is primitive and trivial on units, it follows from the discussions on $[12$, p. $59-60]$ that $\tilde{\chi}$ can be regarded as a primitive Hecke character $\left(\bmod (1+i)^{3} q\right)$ of trivial infinite type. We denote $\tilde{\chi}$ for this Hecke character as well. In the rest of the paper, unless otherwise specified, we shall always regard $\tilde{\chi}$ as a Hecke character.

Let $\psi^{*}(q)$ denote the number of primitive characters $\chi(\bmod q)$ satisfying $\chi(-1)=-1$ and let $\omega(q)$ denote the number of distinct prime ideals dividing $(q)$. Our first result is the following 
Theorem 1.1. For $q \in \mathcal{O}_{K},(q, 2)=1$ and any $\varepsilon>0$, we have, as $N(q) \rightarrow$ $\infty$,

$$
\sum_{\substack{\chi \bmod q \\ \chi(-1)=-1}}^{*} L\left(\frac{1}{2}, \tilde{\chi}\right)=\frac{1}{2} \psi^{*}(q)+O\left(2^{\omega(q)} N(q)^{1 / 2+\varepsilon}\right),
$$

and

$$
\begin{aligned}
& \sum_{\substack{\chi \bmod q \\
\chi(-1)=-1}}^{*}\left|L\left(\frac{1}{2}, \tilde{\chi}\right)\right|^{2} \\
& =\left(\frac{\pi}{16} \frac{\varphi(q)}{N(q)} \log N(q)+\frac{\pi}{8} \frac{\varphi(q)}{N(q)} \sum_{\mathfrak{p} \mid 2 q} \frac{\log N(\mathfrak{p})}{N(\mathfrak{p})-1}+\frac{\varphi(q)}{N(q)} C_{0}\right) \psi^{*}(q) \\
& +O\left(N(q)^{3 / 4+\varepsilon}\right) .
\end{aligned}
$$

Here the $*$ on the sum over $\chi$ restricts the sum to primitive characters, $C_{0}>0$ is an explicitly computable constant and $\varphi(q)=\#\left(\mathcal{O}_{K} /(q)\right)^{*}$.

We note here the asymptotic formulas in Theorem 1.1 are valid for all large $N(q)$ because of the lower bound for $\psi^{*}(q)$ given in $(2.1)$ and if $N(q) \geq$ 3 , then (see $[21,(2.1)])$

$$
\omega(q) \ll \frac{\log N(q)}{\log \log N(q)} .
$$

We readily deduce from Theorem 1.1 via a standard argument using Cauchy's inequality (see $[1$, p. 568]), the following

Corollary 1.2. For $q \in \mathcal{O}_{K},(q, 2)=1$, we have as $N(q) \rightarrow \infty$,

$$
\#\left\{\tilde{\chi}: \chi \bmod q, \chi(-1)=-1, \chi \text { primitive }, L\left(\frac{1}{2}, \tilde{\chi}\right) \neq 0\right\} \gg \frac{\psi^{*}(q)}{\log N(q)} .
$$

Note that Corollary 1.2 does not establish that $L\left(\frac{1}{2}, \widetilde{\chi}\right) \neq 0$ for a positive proportion of the characters $\chi$ to a given modulus. To obtain a positive proportion result, other than studying the mollified moments, we can also study the 1-level densities of low-lying zeros of families of $L$-functions. The density conjecture of N. Katz and P. Sarnak [14, 15] suggests that the distribution of zeros near $1 / 2$ of a family of $L$-functions is the same as that of eigenvalues near 1 of a corresponding classical compact group. This conjecture implies that $L(1 / 2, \chi) \neq 0$ for almost all primitive Dirichlet $L$-functions. Assuming the generalized Riemann hypothesis (GRH), M. R. Murty [18] showed that at least $50 \%$ of both primitive Dirichlet $L$-functions and Dirichlet twists of modular $L$-functions do not vanish at the central point. The result of Murty can be regarded as the 1-level density of low-lying zeros of the corresponding families of $L$-functions for test functions whose Fourier transforms 
being supported in $[-2,2]$. In [11], H. P. Hughes and Z. Rudnick studied the 1-level density of low-lying zeros of the family of primitive Dirichlet $L$-functions of a fixed prime modulus. Their work shows that this family is a unitary family.

Our next result concerns the 1-level density of low-lying zeros of the family $\{L(s, \widetilde{\chi})\}$ of Hecke $L$-functions in $\mathbb{Q}(i)$. Here $\chi$ runs over primitive characters modulo $q$ satisfying $\chi(-1)=-1$ with $q \in \mathbb{Z}[i],(q, 2)=1$. We denote the non-trivial zeroes of the Hecke $L$-function $L(s, \widetilde{\chi})$ by $1 / 2+i \gamma_{\tilde{\chi}, j}$. Without assuming GRH, we order them as

$$
\ldots \leq \Re \gamma_{\widetilde{\chi},-2} \leq \Re \gamma_{\widetilde{\chi},-1}<0 \leq \Re \gamma_{\widetilde{\chi}, 1} \leq \Re \gamma_{\widetilde{\chi}, 2} \leq \ldots
$$

We set

$$
\widetilde{\gamma}_{\tilde{\chi}, j}=\frac{\gamma_{\tilde{\chi}, j}}{2 \pi} \log N(q)
$$

and define for an even Schwartz class function $\phi$,

$$
S(\widetilde{\chi}, \phi)=\sum_{j} \phi\left(\widetilde{\gamma}_{\tilde{\chi}, j}\right) .
$$

Following [11, Definition 2.1], we say a function $f(x)$ is an admissible function if it is a real, even function, whose Fourier transform $\widehat{f}(u)$ is compactly supported, and such that $f(x) \ll(1+|x|)^{-1-\delta}$ for some $\delta>0$. Our result is

Theorem 1.3. Let $\phi(x)$ be an admissible function whose Fourier transform $\widehat{\phi}(u)$ has compact support in $(-2,2)$. Then for $q \in \mathbb{Z}[i],(q, 2)=1$, we have

$$
\lim _{N(q) \rightarrow \infty} \frac{1}{\psi^{*}(q)} \sum_{\substack{\chi \bmod q \\ \chi(-1)=-1}}^{*} S(\tilde{\chi}, \phi)=\int_{\mathbb{R}} \phi(x) \mathrm{d} x .
$$

Here the $*$ on the sum over $\chi$ restricts the sum to primitive characters.

Theorem 1.3 can be regarded as an analogue to the above mentioned result of H. P. Hughes and Z. Rudnick in [11]. The left-hand side expression of (1.5) is known as the 1-level density of low-lying zeros of the family $\{L(s, \widetilde{\chi})\}$. In connection with the random matrix theory (see the discussions in [7]), the right-hand side expression of (1.5) shows that the family is also a unitary family.

Using the argument in the proof of [9, Corollary 1.4], we deduce readily a positive proportion non-vanishing result for the family of Hecke $L$-functions under our consideration. 
Corollary 1.4. Suppose that the GRH is true and that $1 / 2$ is a zero of $L(s, \tilde{\chi})$ of order $n_{\tilde{\chi}} \geq 0$. As $N(q) \rightarrow \infty$,

$$
\sum_{\substack{\chi \bmod q \\ \chi(-1)=-1}}^{*} n_{\tilde{\chi}} \leq\left(\frac{1}{2}+o(1)\right) \psi^{*}(q) .
$$

Moreover, as $N(q) \rightarrow \infty$

$$
\begin{aligned}
\#\left\{\tilde{\chi} \mid \chi \bmod q, \chi(-1)=-1, \chi \text { primitive, } L\left(\frac{1}{2}, \tilde{\chi}\right)\right. & \neq 0\} \\
& \geq\left(\frac{1}{2}+o(1)\right) \psi^{*}(q) .
\end{aligned}
$$

Notations. The following notations and conventions are used throughout the paper.

- $e(z)=\exp (2 \pi i z)=e^{2 \pi i z}$.

- $f=O(g)$ or $f \ll g$ means $|f| \leq c g$ for some unspecified positive constant $c$.

- $f=o(g)$ means $\lim _{x \rightarrow \infty} f(x) / g(x)=0$.

- $K=\mathbb{Q}(i), \mathcal{O}_{K}=\mathbb{Z}[i]$.

- $\mu_{[i]}$ denotes the Möbius function on $\mathcal{O}_{K}$.

- $\varphi$ denotes Euler's totient function on $\mathcal{O}_{K}$.

- $\varpi$ denotes a prime in $K$.

\section{Preliminaries}

2.1. Orthogonality relations and primitive Hecke characters. Let $q \in \mathcal{O}_{K},(q, 2)=1$ and let $\chi$ be a primitive character modulo $q$ defined in (1.1) satisfying $\chi(-1)=-1$. We note the following orthogonality relations. As the proof is similar to the classical case (see [23, Lemma 1]), we omit it here.

Lemma 2.1. Let $q \in \mathcal{O}_{K},(q, 2)=1$. Let $a= \pm 1$, we have for $(n m, q)=1$

$$
\begin{gathered}
\sum_{\substack{\chi \bmod q \\
\chi(-1)=(-1)^{a}}}^{*} \chi(n) \bar{\chi}(m)=\frac{1}{2} \sum_{\substack{d \mid q \\
n \equiv m \bmod d}} \mu_{[i]}(q / d) \varphi(d) \\
+\frac{(-1)^{a}}{2} \sum_{\substack{d \mid q \\
n \equiv-m \bmod d}} \mu_{[i]}(q / d) \varphi(d) .
\end{gathered}
$$

By setting $n=m=1$ in Lemma 2.1, we deduce immediately the following 
Corollary 2.2. Let $q \in \mathcal{O}_{K},(q, 2)=1$ and let $\psi^{*}(q)$ denote the number of primitive characters $\chi(\bmod q)$ satisfying $\chi(-1)=-1$, then

$$
\psi^{*}(q)=\frac{1}{2} \psi(q)-\frac{1}{2} \mu_{[i]}(q),
$$

where $\psi(q)$ denotes the number of primitive characters $\chi(\bmod q)$. Moreover, $\psi(q)$ is a multiplicative function given by $\psi(\varpi)=N(\varpi)-2$ for primes $\varpi$, and $\psi\left(\varpi^{k}\right)=N(\varpi)^{k}(1-1 / N(\varpi))^{2}$ for $k \geq 2$.

We note that Corollary 2.2 implies that for $(q, 2)=1$, we have

$$
\psi^{*}(q), \psi(q) \gg N(q)\left(\frac{\varphi(q)}{N(q)}\right)^{2} \gg \frac{N(q)}{\log \log N(q)} .
$$

Now we show that the induced character $\tilde{\chi}$ modulo $(1+i)^{3} q$ is also primitive. Suppose that $\tilde{\chi}$ is induced by a character modulo $(1+i)^{3} q^{\prime}$ for some proper divisor $q^{\prime}$ of $q$. Then as $\chi$ is primitive, there exists a $c \equiv 1$ $\left(\bmod q^{\prime}\right)$ such that $\chi(c) \neq 1$. By the Chinese Remainder Theorem, we can then find a $c_{0}$ such that $c_{0} \equiv 1\left(\bmod (1+i)^{3}\right)$ and $c_{0} \equiv c(\bmod q)$. It follows from our definition that $\tilde{\chi}\left(c_{0}\right)=\chi(c) \neq 1$. This contradiction shows that $\tilde{\chi}$ can only be possibly induced by a character $\chi^{\prime}$ modulo $(1+i)^{2} q$. But in this case, we can again apply the Chinese Remainder Theorem to find a $c_{0}$ such that $c_{0} \equiv-1\left(\bmod (1+i)^{3}\right)$ and $c_{0} \equiv 1(\bmod q)$. As $-1 \equiv 1$ $\left(\bmod (1+i)^{2}\right)$, we have $c_{0} \equiv 1\left(\bmod (1+i)^{2} q\right)$ so that $\chi^{\prime}\left(c_{0}\right)=1$. However, it follows from the definition that $\widetilde{\chi}\left(c_{0}\right)=\chi\left(-c_{0}\right)=-1$. This implies that $\tilde{\chi}$ can not be induced by $\chi^{\prime}$ either and hence is primitive.

2.2. The approximate functional equation. Let $\tilde{\chi}$ be given as in the previous section regarding as a primitive Hecke character modulo $(1+i)^{3} q$ of trivial infinite type. The Hecke $L$-function associated with this Hecke character $\tilde{\chi}$ is defined for $\Re(s)>1$ by

$$
L(s, \tilde{\chi})=\sum_{0 \neq \mathcal{A} \subset \mathcal{O}_{K}} \tilde{\chi}(\mathcal{A})(N(\mathcal{A}))^{-s},
$$

where $\mathcal{A}$ runs over all non-zero integral ideals in $K$ and $N(\mathcal{A})$ is the norm of $\mathcal{A}$. As shown by E. Hecke, $L(s, \widetilde{\chi})$ admits analytic continuation to an entire function and satisfies a functional equation (see [19, Corollary 8.6]):

$$
\Lambda(s, \tilde{\chi})=g(\widetilde{\chi})\left(N\left((1+i)^{3} q\right)\right)^{-1 / 2} \Lambda(1-s, \overline{\widetilde{\chi}}),
$$

where $D_{K}=-4$ is the discriminant of $K, g(\tilde{\chi})$ is the Gauss sum defined by

$$
g(\tilde{\chi})=\sum_{x \bmod (1+i)^{3} q} \tilde{\chi}(x) \widetilde{e}\left(\frac{x}{(1+i)^{3} q}\right), \quad \widetilde{e}(z)=e\left(\operatorname{tr}\left(\frac{z}{2 i}\right)\right),
$$

and

$$
\Lambda(s, \tilde{\chi})=\left(\left|D_{K}\right| N\left((1+i)^{3} q\right)\right)^{s / 2}(2 \pi)^{-s} \Gamma(s) L(s, \tilde{\chi}) .
$$


We refer the reader to [19] for a more detailed discussion of the Hecke characters and $L$-functions.

Note that we have $|g(\tilde{\chi})|=\left(N\left((1+i)^{3} q\right)\right)^{1 / 2}$ (see $[12$, Exercise 12, p. 61]) and that it follows from the definition that $\overline{g(\widetilde{\chi})}=\widetilde{\chi}(-1) g(\overline{\widetilde{\chi}})=g(\overline{\widetilde{\chi}})$, as $\widetilde{\chi}(-1)(-1)=1$. From this and $(2.2)$, we get that

$$
\Lambda\left(\frac{1}{2}+s, \tilde{\chi}\right) \Lambda\left(\frac{1}{2}+s, \overline{\widetilde{\chi}}\right)=\Lambda\left(\frac{1}{2}-s, \tilde{\chi}\right) \Lambda\left(\frac{1}{2}-s, \overline{\widetilde{\chi}}\right) .
$$

For $c>1 / 2$ we consider

$$
I:=\frac{1}{2 \pi i} \int_{(c)} \frac{\Lambda(1 / 2+s, \tilde{\chi}) \Lambda(1 / 2+s, \overline{\widetilde{\chi}})}{\Gamma(1 / 2)^{2}} \frac{\mathrm{d} s}{s} .
$$

We move the line of integration to $\operatorname{Re}(s)=-c$ and use the relation (2.3) to see that $I=|L(1 / 2, \tilde{\chi})|^{2}-I$, so that $|L(1 / 2, \tilde{\chi})|^{2}=2 I$. On the other hand, expanding $L(1 / 2+s, \widetilde{\chi}) L(1 / 2+s, \overline{\widetilde{\chi}})$ into its Dirichlet series and integrating termwise, we get $I=A(\tilde{\chi})$, where

$$
A(\widetilde{\chi}):=\sum_{0 \neq \mathcal{A}, \mathcal{B} \subset \mathcal{O}_{K}} \tilde{\chi}(\mathcal{A}) \overline{\widetilde{\chi}(\mathcal{B})}(N(\mathcal{A}) N(\mathcal{B}))^{-1 / 2} W\left(\frac{N(\mathcal{A}) N(\mathcal{B})}{N(q)}\right)
$$

with

$$
W(x)=\frac{1}{2 \pi i} \int_{(c)}\left(\frac{\Gamma(s+1 / 2)}{\Gamma(1 / 2)}\right)^{2}\left(\frac{2\left|D_{k}\right|}{\pi^{2}}\right)^{s} x^{-s} \frac{\mathrm{d} s}{s},
$$

for any positive $x, c$. Similar to $[23,(1.3 \mathrm{a}),(1.3 \mathrm{~b})]$, we have for any $j \geq 0$,

$$
W(x)=1+O\left(x^{1 / 2-\varepsilon}\right), \quad W^{(j)}(x)=O_{c}\left(x^{-c}\right) .
$$

On the other hand, we note the following expression for $L(1 / 2, \widetilde{\chi}$ ) (see $[8$, Section 2.3]):

$$
\begin{aligned}
L\left(\frac{1}{2}, \tilde{\chi}\right) & =\sum_{0 \neq \mathcal{A} \subset \mathcal{O}_{K}} \frac{\tilde{\chi}(\mathcal{A})}{N(\mathcal{A})^{1 / 2}} V\left(\frac{N(\mathcal{A})}{x}\right) \\
& +\frac{g(\tilde{\chi})}{N\left((1+i)^{3} q\right)^{1 / 2}} \sum_{0 \neq \mathcal{A} \subset \mathcal{O}_{K}} \frac{\overline{\widetilde{\chi}}(\mathcal{A})}{N(\mathcal{A})^{1 / 2}} V\left(\frac{N(\mathcal{A}) x}{\left|D_{K}\right| N\left((1+i)^{3} q\right)}\right)
\end{aligned}
$$

where $x>0$ and

$$
V(\xi)=\frac{1}{2 \pi i} \int_{(2)} \frac{\Gamma(s+1 / 2)}{\Gamma(1 / 2)} \frac{(2 \pi \xi)^{-s}}{s} \mathrm{~d} s .
$$

We note (see [22, Lemma 2.1]) the following estimation for the $j$-th derivative of $V(\xi)$ :

$$
\begin{aligned}
& V(\xi)=1+O\left(\xi^{1 / 2-\varepsilon}\right) \text { for } 0<\xi<1 \\
& \qquad \text { and } V^{(j)}(\xi)=O\left(e^{-\xi}\right) \text { for } \xi>0, j \geq 0 .
\end{aligned}
$$


2.3. The explicit formula. Our approach of Theorem 1.3 relies on the following explicit formula, which essentially converts a sum over zeros of an $L$-function to a sum over primes. As it is similarly to that of [7, Lemma 2.3], we omit its proof here.

Lemma 2.3. Let $\phi(x)$ be an admissible function whose Fourier transform $\widehat{\phi}(u)$ has compact support in $[-2,2]$. Let $\Lambda_{K}$ be the von Mangoldt function in $K$. Then for $q \in \mathcal{O}_{K},(q, 2)=1$ and any primitive character $\chi$ modulo $q$ satisfying $\chi(-1)=-1$, we have

$$
\begin{array}{r}
S(\tilde{\chi}, \phi)=\int_{-\infty}^{\infty} \phi(t) \mathrm{d} t-\frac{1}{\log X} \sum_{(n)} \frac{\Lambda_{K}(n)}{\sqrt{N(n)}} \widehat{\phi}\left(\frac{\log N(n)}{\log N(q)}\right)(\tilde{\chi}(n)+\overline{\widetilde{\chi}}(n)) \\
+O\left(\frac{1}{\log N(q)}\right) .
\end{array}
$$

\section{Proof of Theorem 1.1}

3.1. Evaluation of the first moment. Since any integral non-zero ideal $\mathcal{A}$ co-prime to 2 in $\mathcal{O}_{K}$ has a unique primary generator $a$, we apply the approximate functional equation (2.6) and the orthogonality relations Lemma 2.1 to get that

$$
\begin{aligned}
& \sum_{\substack{\chi \bmod q \\
\chi(-1)=-1}}^{*} L\left(\frac{1}{2}, \tilde{\chi}\right) \\
& =\sum_{n \equiv 1 \bmod (1+i)^{3}} \frac{1}{\sqrt{N(n)}} V\left(\frac{N(n)}{x}\right) \sum_{\substack{\chi \bmod q \\
\chi(-1)=-1}}^{*} \tilde{\chi}(n) \\
& \quad+\frac{1}{(8 N(q))^{1 / 2}} \sum_{n \equiv 1 \bmod (1+i)^{3}} \frac{1}{\sqrt{N(n)}} V\left(\frac{N(n) x}{32 N(q)}\right) \sum_{\substack{\chi \bmod q \\
\chi(-1)=-1}}^{*} \overline{\widetilde{\chi}}(n) g(\tilde{\chi}) \\
& =S_{1,1}+S_{1,2}+S_{1,3}+S_{1,4},
\end{aligned}
$$

where

$$
\begin{aligned}
S_{1,1} & =\frac{1}{2} \sum_{\substack{d \mid q \\
d \equiv 1 \bmod (1+i)^{3}}} \mu_{[i]}(q / d) \varphi(d) \sum_{\substack{n \equiv 1 \bmod (1+i)^{3} d \\
(n, q)=1}} \frac{1}{\sqrt{N(n)}} V\left(\frac{N(n)}{x}\right), \\
S_{1,2} & =-\frac{1}{2} \sum_{\substack{d \mid q \\
d \equiv 1 \bmod (1+i)^{3}}} \mu_{[i]}(q / d) \varphi(d) \sum_{\substack{n \equiv-1 \bmod d \\
n \equiv 1 \bmod (1+i)^{3} \\
(n, q)=1}} \frac{1}{\sqrt{N(n)}} V\left(\frac{N(n)}{x}\right),
\end{aligned}
$$




$$
\begin{gathered}
S_{1,3}=\frac{1}{2} \cdot \frac{1}{(8 N(q))^{1 / 2}} \sum_{d \mid q} \mu_{[i]}(q / d) \varphi(d) \sum_{n \equiv 1 \bmod (1+i)^{3}} \frac{1}{\sqrt{N(n)}} \\
\quad \times V\left(\frac{N(n) x}{32 N(q)}\right) \sum_{\substack{x \bmod (1+i)^{3} q \\
x \equiv n \bmod d}} \tilde{e}\left(\frac{x}{(1+i)^{3} q}\right) \\
S_{1,4}=-\frac{1}{2} \cdot \frac{1}{(8 N(q))^{1 / 2}} \sum_{d \mid q} \mu_{[i]}(q / d) \varphi(d) \sum_{n \equiv 1 \bmod (1+i)^{3}} \frac{1}{\sqrt{N(n)}} \\
\quad \times V\left(\frac{N(n) x}{32 N(q)} \sum_{\substack{d \equiv 1 \bmod (1+i)^{3} \\
x \equiv-n \bmod d}} \tilde{e}\left(\frac{x}{(1+i)^{3} q}\right)\right.
\end{gathered}
$$

As $\widetilde{e}(c) \ll 1$ for $c \in \mathcal{O}_{K}$, we have that

$$
\sum_{\substack{x \bmod (1+i)^{3} q \\ x \equiv n \bmod d}} \tilde{e}\left(\frac{x}{(1+i)^{3} q}\right) \ll \sum_{\substack{x \bmod (1+i)^{3} q \\ x \equiv n \bmod d}} 1 \ll \frac{N(q)}{N(d)} .
$$

It follows that

$$
\begin{aligned}
S_{1,3} & \ll N(q)^{1 / 2} \sum_{d \mid q} \mu_{[i]}^{2}(q / d) \frac{\varphi(d)}{N(d)} \\
\times & \sum_{n \equiv 1 \bmod (1+i)^{3}} \frac{1}{\sqrt{N(n)}(1+i)^{3}} V\left(\frac{N(n) x}{32 N(q)}\right) \\
& \ll \frac{N(q)^{1+\varepsilon}}{x^{1 / 2}} 2^{\omega(q)} .
\end{aligned}
$$

Similarly, we also have

$$
S_{1,4} \ll \frac{N(q)^{1+\varepsilon}}{x^{1 / 2}} 2^{\omega(q)} .
$$

In the evaluation of $S_{1,1}$, we write $n=t d+1$ with $t \in \mathcal{O}_{K}$. The term $t=0$ gives the main term:

$$
\begin{aligned}
M_{1} & =\frac{1}{2} \sum_{\substack{d \mid q \\
d \equiv 1 \bmod (1+i)^{3}}} \mu_{[i]}(q / d) \varphi(d) V\left(\frac{1}{x}\right) \\
& =\frac{1}{2} \sum_{d \mid q} \mu_{[i]}(q / d) \varphi(d)\left(1+O\left(x^{-1 / 2+\varepsilon}\right)\right) \\
& =\frac{1}{2} \psi^{*}(q)+O\left(N(q) x^{-1 / 2+\varepsilon}\right),
\end{aligned}
$$


where we have used Corollary 2.2 and the fact that

$$
\sum_{\substack{d \mid q \\ d \equiv 1 \bmod (1+i)^{3}}} \varphi(d)=N(q) .
$$

To treat the contribution from the terms $n \neq 1$ in $S_{1,1}$, we need the following lemma.

Lemma 3.1. Let $m, n \in \mathbb{Z}[i]$ satisfying $N(m+n) \geq N(n)$, then we have

$$
N(m+n) \geq \frac{N(m)}{64} \text {. }
$$

Proof. The assertion of the Lemma is clearly true when $N(n) \geq \frac{N(m)}{64}$. We may therefore assume that $N(n) \leq \frac{N(m)}{64}$. Writing $m=a+b i, n=c+d i$ with $a, b, c, d \in \mathbb{Z}$, we see that $N(n) \leq \frac{N(m)}{64}$ is equivalent to

$$
\frac{a^{2}+b^{2}}{64} \geq c^{2}+d^{2} \text {. }
$$

We deduce from this that

$$
\max \{|c|,|d|\} \leq \frac{\sqrt{a^{2}+b^{2}}}{8} .
$$

Writing (3.2) in terms of $a, b, c, d$, we find that it suffices to show

$$
a^{2}+2 a c+b^{2}+2 b d \geq \frac{a^{2}+b^{2}}{64} .
$$

Applying (3.3), we see that

$$
a^{2}+2 a c+b^{2}+2 b d \geq a^{2}+b^{2}-(|a|+|b|) \frac{\sqrt{a^{2}+b^{2}}}{4} .
$$

As the above inequality implies inequality (3.4), the assertion of the lemma now follows.

Applying Lemma 3.2 to the case $n=t d+1$ with $t \neq 0$, we see that in this case $N(t d+1) \geq N(t d) / 64$. In view of the rapid decay of $V$ in (2.7), we may further assume that $N(n) \leq x^{1+\varepsilon}$ for any $\varepsilon>0$. This implies that $N(t d) \leq 64 x^{1+\varepsilon}$. We then deduce that the terms with $t \neq 0$ in $S_{1,1}$ contribute an amount that is

$$
\ll \sum_{\substack{d \mid q \\ d \equiv 1 \bmod (1+i)^{3}}} \mu_{[i]}^{2}(q / d) \varphi(d) \sum_{0 \neq N(t d) \leq 64 x^{1+\varepsilon}} \frac{1}{\sqrt{N(t d)}} \ll 2^{\omega(q)} x^{1 / 2+\varepsilon} .
$$

Thus, we have

$$
S_{1,1}=\frac{1}{2} \psi^{*}(q)+O\left(N(q) x^{-1 / 2+\varepsilon}+2^{\omega(q)} x^{1 / 2+\varepsilon}\right) .
$$


Now, to estimate $S_{1,2}$, we write $n=t d-1$ with $t \in \mathcal{O}_{K}$. Note that in this case $t \neq 0$ since -1 is not primitive. The treatment of the contribution from these $t \neq 0$ terms is similar to that of $S_{1,1}$ and we arrive at

$$
S_{1,2} \ll 2^{\omega(q)} x^{1 / 2+\varepsilon} .
$$

We then conclude that

$$
\begin{aligned}
& \sum_{\substack{\chi \bmod q \\
\chi(-1)=-1}}^{*} L\left(\frac{1}{2}, \tilde{\chi}\right) \\
& \quad=\frac{1}{2} \psi^{*}(q)+O\left(N(q) x^{-1 / 2+\varepsilon}+2^{\omega(q)} x^{1 / 2+\varepsilon}+\frac{N(q)^{1+\varepsilon}}{x^{1 / 2}} 2^{\omega(q)}\right) .
\end{aligned}
$$

By setting $x=N(q)$, we obtain (1.2).

3.2. The main term of the second moment. To establish (1.3), we note that it is shown in Section 2.2 that $|L(1 / 2, \widetilde{\chi})|^{2}=2 A(\widetilde{\chi})$ with $A(\widetilde{\chi})$ given in (2.4). Again writing any integral non-zero ideal $\mathcal{A}$ co-prime to 2 in $\mathcal{O}_{K}$ in term of its unique primary generator $a$ and applying Lemma 2.1, we have

$$
\begin{aligned}
\sum_{\substack{\chi \bmod q \\
\chi(-1)=-1}}^{*} & |L(1 / 2, \tilde{\chi})|^{2} \\
& =2 \sum_{\substack{n, m \\
n, m \text { primary }}} \frac{1}{\sqrt{N(n) N(m)}} W\left(\frac{N(n m)}{N(q)}\right) \sum_{\substack{\chi \bmod q \\
\chi(-1)=-1}}^{*} \tilde{\chi}(n) \bar{\chi}(m) \\
& =S_{2,1}-S_{2,2},
\end{aligned}
$$

where

$$
\begin{aligned}
S_{2,1} & =\sum_{\substack{d \mid q \\
d \text { primary }}} \mu_{[i]}(d) \varphi(q / d) \sum_{\substack{n \equiv m \bmod q / d \\
n, m \text { primary } \\
(m n, q)=1}} \frac{1}{\sqrt{N(n) N(m)}} W\left(\frac{N(n m)}{N(q)}\right), \\
S_{2,2} & =\sum_{\substack{d \mid q \\
d \text { primary }}} \mu_{[i]}(d) \varphi(q / d) \sum_{\substack{n \equiv-m \text { mod } q / d \\
n, m \text { primary } \\
(m n, q)=1}} \frac{1}{\sqrt{N(n) N(m)}} W\left(\frac{N(n m)}{N(q)}\right) .
\end{aligned}
$$

We consider the terms $n=m$ in $S_{2,1}$. These terms contribute

$$
M_{2}=\sum_{\substack{d \mid q \\ d \text { primary }}} \mu_{[i]}(d) \varphi(q / d) \sum_{\substack{n \text { primary } \\(n, q)=1}} \frac{1}{N(n)} W\left(\frac{N(n)^{2}}{N(q)}\right) .
$$


We then apply Mellin inversion to get

$$
\begin{aligned}
& \sum_{\substack{n \text { primary } \\
(n, q)=1}} \frac{1}{N(n)} W\left(\frac{N(n)^{2}}{N(q)}\right) \\
& \quad=\frac{1}{2 \pi i} \int_{(2)} \sum_{\substack{n \text { primary } \\
(n, q)=1}} \frac{1}{N(n)^{1+2 s}} N(q)^{s} \widehat{W}(s) \mathrm{d} s \\
& \quad=\frac{1}{2 \pi i} \int_{(2)} \zeta_{K}(1+2 s)\left(\prod_{\mathfrak{p} \mid 2 q}\left(1-N(\mathfrak{p})^{-(1+2 s)}\right)\right) N(q)^{s} \widehat{W}(s) \mathrm{d} s .
\end{aligned}
$$

Here and in what follows, we use $\zeta_{K}(s)$ to denote the Dedekind zeta function of $K$ and $\mathfrak{p}$ to denote prime ideals in $\mathcal{O}_{K}$. Moreover, $\widehat{W}(s)$ is the Mellin transform of $W(t)$, so that

$$
\widehat{W}(s)=\int_{0}^{\infty} W(t) t^{s} \frac{\mathrm{d} t}{t} .
$$

Using (2.5) and integration by parts implies that for $\Re(s)>0$,

$$
\widehat{W}(s)=\frac{1}{s} I(s), \quad I(s)=\int_{0}^{\infty} W^{\prime}(t) t^{s} \mathrm{~d} t .
$$

Note that (2.5) further implies that $I(0)=1$ and integration by parts implies that $I(s)$ is clearly analytic for $\Re(s)>-1$ and satisfies

$$
I(s) \ll \frac{1}{|1+s|} .
$$

It follows that (3.6) gives an analytic extension of $\widehat{W}(s)$ to $\Re(s)>-1$ with a simple pole at $s=0$ with residue 1 such that when $\Re(s)>-1$,

$$
\widehat{W}(s) \ll \frac{1}{|s||1+s|} .
$$

We now shift the line of integration in (3.5) to $\Re(s)=-1 / 4+\varepsilon$ and we encounter a double pole at $s=0$. The residue is easily seen (by taking note that the residue of $\zeta_{K}(s)$ at $s=1$ is $\left.\pi / 4\right)$ to be

$$
\frac{\pi}{16} \frac{\varphi(q)}{N(q)} \log N(q)+\frac{\pi}{8} \frac{\varphi(q)}{N(q)} \sum_{\mathfrak{p} \mid 2 q} \frac{\log N(\mathfrak{p})}{N(\mathfrak{p})-1}+\frac{\varphi(q)}{N(q)} C_{0}
$$

where $C_{0}$ is an explicitly computable positive constant.

Since $\sum_{\mathfrak{p} \mid q} \frac{\log N(\mathfrak{p})}{N(\mathfrak{p})-1}$ is the largest when $q$ is of the form $\prod_{N(\varpi) \leq y} \varpi$ for primes $\varpi$, it follows from this and the prime ideal theorem [17, Theorem 8.9] 
that

$$
\sum_{\mathfrak{p} \mid q} \frac{\log N(\mathfrak{p})}{N(\mathfrak{p})-1} \ll 1+\log \omega(q) .
$$

To estimate the remaining integral at $\Re(s)=-1 / 4+\varepsilon$, we shall use the convexity bound that (see [12, Exercise 3, p. 100]) for $\Re(s)=-1 / 4+\varepsilon$,

$$
\zeta_{K}(1+2 s) \ll\left(1+|s|^{2}\right)^{1 / 4+\varepsilon} .
$$

Applying this and (3.7) gives that the integral on the line $\Re(s)=-1 / 4+\varepsilon$ is $\ll N(q)^{-1 / 4+\varepsilon}$. From this and (3.8), we get

$$
\begin{aligned}
M_{2}=\left(\frac{\pi}{16}\right. & \left.\frac{\varphi(q)}{N(q)} \log N(q)+\frac{\pi}{8} \frac{\varphi(q)}{N(q)} \sum_{\mathfrak{p} \mid 2 q} \frac{\log N(\mathfrak{p})}{N(\mathfrak{p})-1}+\frac{\varphi(q)}{N(q)} C_{0}\right) \\
& \times \sum_{\substack{d \mid q \\
d \text { primary }}} \mu_{[i]}(d) \varphi(q / d)+O\left(N(q)^{-1 / 4+\varepsilon} \sum_{d \mid q} \mu_{[i]}^{2}(d) \varphi(q / d)\right) .
\end{aligned}
$$

We then deduce using (3.1) and (3.9) that

$$
\begin{array}{r}
M_{2}=\left(\frac{\pi}{16} \frac{\varphi(q)}{N(q)} \log N(q)+\frac{\pi}{8} \frac{\varphi(q)}{N(q)} \sum_{\mathfrak{p} \mid 2 q} \frac{\log N(\mathfrak{p})}{N(\mathfrak{p})-1}\right. \\
\left.+\frac{\varphi(q)}{N(q)} C_{0}\right) \psi^{*}(q) \\
+O\left(N(q)^{3 / 4+\varepsilon}\right)
\end{array}
$$

3.3. The error term of the second moment. We first note that the terms $n=m$ in $S_{2,2}$ can occur if and only if $2 n \equiv 0(\bmod q / d)$. As $(q, 2)=$ 1 , this occurs if and only if $q / d \mid n$. It follows readily from this that the terms $n=m$ in $S_{2,2}$ contribute

$$
\ll 2^{\omega(q)} \log N(q) .
$$

To treat the contributions from the terms $n \neq m$ in $S_{2,1}$ and $S_{2,2}$, we note the following

Lemma 3.2. We have for any $\varepsilon>0$,

$$
\sum_{\substack{n \neq m \\ n=m \bmod \ell \\(n m, q)=1}} \frac{1}{\sqrt{N(n) N(m)}} W\left(\frac{N(n m)}{N(q)}\right) \ll \frac{N(q)^{1 / 2+\varepsilon}}{N(l)} .
$$

Proof. We may assume that $N(m) \geq N(n)$. In view of the rapid decey of $W$ shown in (2.5), we may further assume that $N(n m) \leq N(q)^{1+\varepsilon}$ for any 
$\varepsilon>0$. We then have

$$
\begin{aligned}
\sum_{\substack{n \neq m \\
n \equiv m \bmod \ell \\
(n m, q)=1}} \frac{1}{\sqrt{N(n) N(m)}} W\left(\frac{N(n m)}{N(q)}\right) & \\
& \ll \sum_{N(n) \leq N(q)^{1+\varepsilon}} \frac{1}{\sqrt{N(n)}} \sum_{\substack{m \neq n \\
m \equiv n \bmod \ell \\
N(n) \leq N(m) \leq N(q)^{1+\varepsilon} / N(n)}} \frac{1}{\sqrt{N(m)}} .
\end{aligned}
$$

We write $m=n+k l$ with $k \in \mathcal{O}_{K}$ and we apply Lemma 3.1 to see that $N(k l) \leq 64 N(m) \leq 64 N(q)^{1+\varepsilon} / N(n)$. Thus, we have

$$
\begin{aligned}
\sum_{\substack{m \neq n \\
m \equiv n \bmod \ell \\
N(n) \leq N(m) \leq N(q)^{1+\varepsilon / N(n)}}} \frac{1}{\sqrt{N(m)}} & \ll \frac{1}{\sqrt{N(l)}} \sum_{0 \neq N(k) \leq 64 N(q)^{1+\varepsilon / N(n)}} \frac{1}{\sqrt{N(k l)}} \\
& \ll \frac{1}{N(l) \sqrt{N(n)}} N(q)^{1 / 2+\varepsilon} .
\end{aligned}
$$

Applying this in (3.13), we readily deduce (3.12) and this completes the proof of the lemma.

It follows from Lemma 3.2 that the terms $n \neq m$ contribute in $S_{2,1}, S_{2,2}$

$$
\ll 2^{\omega(q)} N(q)^{1 / 2+\varepsilon} \text {. }
$$

Using (1.4) and combining (3.10), (3.11) and (3.14), the proof of (1.3) is complete.

\section{Proof of Theorem 1.3}

Applying Lemma 2.3, we see that it suffices to show that for any $\widehat{\phi}$ supported in $(-2+\varepsilon, 2-\varepsilon)$ with any $0<\varepsilon<1$,

$$
\lim _{N(q) \rightarrow \infty} \frac{\widetilde{S}(q, \widehat{\phi})}{N(q) \log N(q)}=0
$$

where

$$
\widetilde{S}(q, \widehat{\phi})=\sum_{\substack{\chi \bmod q \\ \chi(-1)=-1}}^{*} \sum_{\text {primary }} \frac{\Lambda_{K}(n)}{\sqrt{N(n)}} \widehat{\phi}\left(\frac{\log N(n)}{\log N(q)}\right)(\tilde{\chi}(n)+\overline{\widetilde{\chi}}(n)) .
$$


Applying Lemma 2.1, we see that

$$
\begin{aligned}
\widetilde{S}(q, \widehat{\phi})= & \sum_{\substack{d \mid q \\
d \equiv 1 \bmod (1+i)^{3}}} \mu_{[i]}(q / d) \varphi(d) \sum_{\substack{n \text { primary } \\
n \equiv 1 \bmod d}} \frac{\Lambda_{K}(n)}{\sqrt{N(n)}} \widehat{\phi}\left(\frac{\log N(n)}{\log q}\right) \\
& -\sum_{\substack{d \mid q \\
d \equiv 1 \bmod (1+i)^{3}}} \mu_{[i]}(q / d) \varphi(d) \sum_{\substack{n \text { primary } \\
n \equiv-1 \bmod d}} \frac{\Lambda_{K}(n)}{\sqrt{N(n)}} \widehat{\phi}\left(\frac{\log N(n)}{\log q}\right) .
\end{aligned}
$$

Similar to the treatment of the case $n \neq 1$ in $S_{1,1}$ in Section 3.1, we have

$$
\begin{aligned}
\sum_{\substack{n \text { primary } \\
n \equiv \pm 1 \text { mod } d}} \frac{\Lambda_{K}(n)}{\sqrt{N(n)}} \widehat{\phi}\left(\frac{\log N(n)}{\log q}\right) & \ll \sum_{\substack{n \text { primary } \\
1<N(n) \leq q^{2-\varepsilon} \\
n \equiv \pm 1 \text { mod } d}} \frac{\log N(q)}{\sqrt{N(n)}} \\
& \ll \frac{N(q)^{1-\varepsilon / 2} \log N(q)}{N(d)} .
\end{aligned}
$$

It follows that

$$
\begin{aligned}
\widetilde{S}(q, \widehat{\phi}) & \ll \sum_{\substack{d \mid q \\
d \equiv 1 \bmod (1+i)^{3}}} \mu_{[i]}^{2}(q / d) \varphi(d) \frac{N(q)^{1-\varepsilon / 2} \log N(q)}{N(d)} \\
& \ll 2^{\omega(q)} N(q)^{1-\varepsilon / 2} \log N(q) .
\end{aligned}
$$

In view of (1.4), the desired limit in (4.1) follows from the above estimation and this completes the proof of Theorem 1.3.

\section{References}

[1] R. Balasubramanian \& V. K. Murty, "Zeros of Dirichlet L-functions", Ann. Sci. Éc. Norm. Supér. 25 (1992), no. 5, p. 567-615.

[2] B. C. Berndt, R. J. Evans \& K. S. Williams, Gauss and Jacobi sums, Canadian Mathematical Society Series of Monographs and Advanced Texts, John Wiley \& Sons, 1998.

[3] V. Blomer, É. Fouvry, E. Kowalski, P. Michel \& D. Milićević, "On moments of twisted L-functions", Am. J. Math. 139 (2017), no. 3, p. 707-768.

[4] H. M. Bui, "Non-vanishing of Dirichlet $L$-functions at the central point", Int. J. Number Theory 8 (2012), no. 8, p. 1855-1881.

[5] S. ChOwla, The Riemann hypothesis and Hilbert's tenth problem, Mathematics and its Applications, vol. 4, Gordon and Breach Science Publishers, 1965.

[6] P. Gao, R. Khan \& G. Ricotta, "The second moment of Dirichlet twists of Hecke $L$ functions", Acta Arith. 140 (2009), no. 1, p. 57-65.

[7] P. Gao \& L. Zhaо, "One level density of low-lying zeros of families of $L$-functions", Compos. Math. 147 (2011), no. 1, p. 1-18.

[8] - "Moments of Quadratic Hecke L-functions of Imaginary Quadratic Number Fields", J. Number Theory 209 (2020), p. 359-377.

[9] - "One level density of low-lying zeros of quadratic and quartic Hecke $L$-functions", Can. J. Math. 72 (2020), no. 2, p. 427-454.

[10] D. R. HeAth-Brown, "The fourth power mean of Dirichlet's L-functions", Analysis 1 (1981), no. 1 , p. 25-32. 
[11] C. P. Hughes \& Z. Rudnick, "Linear statistics of low-lying zeros of $L$-functions", $Q . J$. Math 54 (2003), no. 3, p. 309-333.

[12] H. Iwaniec \& E. Kowalski, Analytic Number Theory, Colloquium Publications, vol. 53, American Mathematical Society, 2004.

[13] H. Iwaniec \& P. Sarnak, "Dirichlet L-functions at the central point", in Number theory in progress. Volume 2: Elementary and analytic number theory, Walter de Gruyter, 1999, p. $941-952$.

[14] N. Katz \& P. SARnak, Random matrices, Frobenius eigenvalues, and monodromy, Colloquium Publications, vol. 45, American Mathematical Society, 1999.

[15] - "Zeroes of zeta functions and symmetries", Bull. Am. Math. Soc. 36 (1999), no. 1, p. $1-26$.

[16] R. Khan \& H. T. NGo, "Nonvanishing of Dirichlet L-functions", Algebra Number Theory 10 (2016), no. 10, p. 2081-2091.

[17] H. L. Montgomery \& R. C. Vaughan, Multiplicative number theory. I. Classical theory, Cambridge Studies in Advanced Mathematics, vol. 97, Cambridge University Press, 2007.

[18] M. R. MurTy, "On simple zeros of certain L-series", in Number theory (Banff, 1988), Walter de Gruyter, 1990, p. 427-439.

[19] J. Neukirch, Algebraic number theory, Grundlehren der Mathematischen Wissenschaften, vol. 322, Springer, 1999.

[20] R. E. A. C. PAley, "On the $k$-analogues of some theorems in the theory of the Riemann ל-function", Proc. Lond. Math. Soc. 32 (1931), p. 273-311.

[21] W. SchaAL, "Obere und untere Abschätzungen in algebraischen Zahlkörpern mit Hilfe des linearen Selbergschen Siebes", Acta Arith. 13 (1968), p. 267-313.

[22] K. Soundararajan, "Nonvanishing of quadratic Dirichlet $L$-functions at $s=\frac{1}{2}$ ", Ann. Math. 152 (2000), no. 2, p. 447-488.

[23] - "The fourth moment of Dirichlet L-functions", in Analytic Number Theory, Clay Mathematics Proceedings, vol. 7, American Mathematical Society, 2007, p. 239-246.

[24] T. Stefanicki, "Non-vanishing of $L$-functions attached to automorphic representations of GL(2) over Q", J. Reine Angew. Math. 474 (1996), p. 1-24.

[25] M. P. Young, "The fourth moment of Dirichlet L-functions", Ann. Math. 173 (2011), no. 1, p. $1-50$.

Peng GaO

School of Mathematical Sciences

Beihang University

Beijing 100191, China

E-mail: penggao@buaa.edu.cn

Liangyi ZHAO

School of Mathematics and Statistics

University of New South Wales

Sydney NSW 2052, Australia

E-mail: 1.zhao@unsw.edu.au

URL: https://web.maths. unsw.edu.au/ leezhao/ 